\title{
Instrumente einer gestaltenden Strukturpolitik?
}

\author{
Überlegungen zum 28. Subventionsbericht 2021
}

\begin{abstract}
Während der Corona-Krise tätigte der Staat hohe Ausgaben, die die wirtschaftliche Entwicklung stabilisieren sollten. Langfristig dienen Subventionen eher dazu, Unternehmen bei der Sicherung ihrer Wettbewerbsfähigkeit zu unterstützen, Forschung und Investitionen zu erhöhen und damit auch den Strukturwandel voranzutreiben. Allerdings werden Subventionen kritisch betrachtet: Einerseits weisen sie Potenzial für die Kürzung öffentlicher Ausgaben auf, andererseits sollen sie Beiträge zu einem nachhaltig ausgerichteten Strukturwandel leisten. Dies ist im Folgenden Anlass dafür, die im 28. Subventionsbericht ausgewiesenen Subventionen kritisch zu beleuchten. Dabei stehen nicht die Corona-Hilfen im Blickpunkt, sondern längerfristig begründete Subventionen, welche die finanziellen Spielräume des Staates dauerhaft einengen.
\end{abstract}

\begin{abstract}
Die Subventionsberichte der Bundesregierung zeigen „komplexe Subventionslandschaften“. Im 28. Bericht (2021) werden 128 verschiedene Finanzhilfen und 106 Steuervergünstigungen ausgewiesen, die sich insgesamt im Jahr 2022 auf 47,2 Mrd. Euro (nach 2019: 24,6 Mrd. Euro) belaufen werden (BMF, 2021, 6 und Anhänge 7 und 8). Diese Zahlen spiegeln teilweise den Anstieg während der Corona-Pandemie in den Jahren 2020/2021 wider. Der Anteil der Finanzhilfen des Bundes an den Ausgaben des Bundes lag 2020 bei 2,6\%, der der Steuervergünstigungen an den Steuereinnahmen bei ca. $6 \%$. (BMF, 2021, 6, sowie Anhänge 7 und 8). Insgesamt handelt es sich um erhebliche Ausgaben mit vielschichtigen langfristigen Wirkungen.
\end{abstract}

\section{Umfang und Definitionen von Subventionen}

Subventionen sind Unterstützungsleistungen des Staates oder von Institutionen der EU an Unternehmen oder

(c) Der/die Autor:in 2022. Open Access: Dieser Artikel wird unter der Creative Commons Namensnennung 4.0 International Lizenz veröffentlicht (creativecommons.org/licenses/by/4.0/deed.de).

Open Access wird durch die ZBW - Leibniz-Informationszentrum Wirtschaft gefördert.

Prof. Dr. Ricarda Kampmann lehrt Volkswirtschaftslehre an der Westfälischen Hochschule in Gelsenkirchen. an private Haushalte, denen keine marktliche Gegenleistung der Empfänger:innen gegenübersteht. Die häufigsten Subventionsformen sind Finanzhilfen und Steuervergünstigungen. Finanzhilfen sind finanzielle Zuschüsse an einzelne Unternehmen, Unternehmen einer bestimmten Branche oder an strukturschwache Regionen. Steuervergünstigungen entlasten von der regulären Steuerpflicht - etwa bei der Mehrwert- oder Energiesteuer. Auch bestimmte Zahlungen an private Haushalte mit branchenspezifischer Wirkung (z. B. Wohnungsbauprämien) zählen zu den Subventionen.

In der Literatur finden sich verschiedene Subventionsbegriffe. Der enge Begriff der Subventionsberichte der Bundesregierung bezieht die vom Bund an Stellen auBerhalb der Bundesverwaltung gezahlten Subventionen ein. Damit bleiben z. B. Subventionen der Bundesländer und der EU zunächst unberücksichtigt, sie werden in den Subventionsberichten aber nachrichtlich ausgewiesen (vgl. Tabelle 1). Zudem werden Zahlungen der Bundesregierung an Stellen innerhalb der Bundesverwaltung nicht erfasst und Zahlungen für „allgemeine Staatsaufgaben“ z. B. für die „Aufrechterhaltung der öffentlichen Ordnung“ (BMF, 2021, 13) oder für Grundlagenforschung - werden hier nicht als Subvention angesehen. In dieser Abgrenzung lagen die Finanzhilfen und Steuervergünstigungen des Bundes im Jahr 2020 bei ca. $40 \%$ des nachrichtlich ausgewiesenen Gesamtvolumens (vgl. Tabelle 1).

Im Gegensatz dazu legt das Kieler Institut für Weltwirtschaft regelmäßig Subventionsberichte vor, in denen der Subventionsbegriff sehr viel weiter gefasst wird. Hier werden beispielsweise Zahlungen der Bundesländer, der 
Tabelle 1

Erfasstes Gesamtvolumen der Subventionen von Bund, Ländern und Gemeinden

European Recovery Program (ERP) und EU in Mrd. Euro

\begin{tabular}{lrrrr} 
& 2000 & 2018 & 2019 & 2020 \\
\hline 1. Finanzhilfen & & & & \\
\hline Bund & 10,1 & 6,7 & 8,3 & 11,7 \\
\hline Länder $^{1}$ & 11,2 & 9,2 & 10,0 & 17,7 \\
\hline Gemeinden $^{2}$ & 1,6 & 3,0 & 3,2 & 3,0 \\
\hline 2. Steuervergünstigungen & & & & \\
\hline Bund & 13,1 & 16,0 & 16,3 & 15,4 \\
\hline Länder, Gemeinden $^{3}$ & 12,0 & 15,7 & 14,4 & 14,0 \\
\hline 3. ERP-Finanzhilfen & 5,7 & 0,2 & 0,2 & 0,2 \\
\hline 4. Marktordnungsausgaben der EU & 5,6 & 5,0 & 4,9 & 4,9 \\
\hline Gesamtvolumen & 59,4 & 55,8 & 57,3 & 66,9 \\
\hline $\begin{array}{l}\text { Anteil Subventionen des Bundes am } \\
\text { ausgewiesenen Gesamtvolumen (in \%) }\end{array}$ & 39,1 & 40,7 & 42,9 & 40,1 \\
\hline
\end{tabular}

${ }^{1}$ Quelle: Zentrale Datenstelle der Landesfinanzminister (ZDL). ${ }^{2}$ Daten der Gemeinden: Schätzung auf der Grundlage der Subventionsentwicklung der Länder; ab 2010 Quelle: ZDL. ${ }^{3}$ Siehe auch Übersicht 7 dieses Berichts. Ab 2008: Umstellung vom Darlehensvolumen auf die gewährten Zinsverbilligungen, die mit den Zusagen des aktuellen Jahrs über die Laufzeit des Kredits bzw. anderer Finanzierungsmaßnahmen verbunden sind.

Quelle: BMF $(2021,39)$

Gemeinden und der EU sowie der Bundesagentur für Arbeit einbezogen. Darüber hinaus zielt diese Definition der Subventionen darauf ab, alle Maßnahmen zu erfassen, die nicht unmittelbar als Staatsaufgaben anzusehen sind und deshalb marktwirtschaftlich erfüllt werden könnten (Boss und Rosenschon, 2006, 6).

Dieser Auffassung zufolge handelt es sich nur dann nicht um Subventionen, wenn der Staat öffentliche Güter anbietet, die nach den Kriterien Nicht-Rivalität im Konsum und Nicht-Ausschlussmöglichkeit zahlungsunwilliger Kund:innen abgegrenzt werden. Nicht unter den Subventionsbegriff fällt hier z. B. der Einsatz öffentlicher Mittel im Ausbildungsbereich und - analog zur Definition des Bundes - in der Grundlagenforschung, weil positive externe Effekte Ausnahmebereiche vom Markt rechtfertigen. Zuschüsse an halbstaatliche Organisationen wie beispielsweise Gesetzliche Krankenversicherungen, Krankenhäuser, Kindergärten, Kirchen, Theater, Museen oder Freizeiteinrichtungen stellen nach der Kieler Definition Subventionen dar (Laaser und Rosenschon, 2020, 57).

Meritorische Güter, die der Staat anbietet, um eine höhere Inanspruchnahme dieser Güter zu ermöglichen, könnten demnach - vermutlich zu höheren Preisen und daher in geringerer Menge - prinzipiell über Märkte angeboten
Tabelle 2

Vergleich der Volumina der Finanzhilfen des Bundes in verschiedenen Abgrenzungen

in Mio. Euro

\begin{tabular}{|c|c|c|c|c|c|c|}
\hline & 2000 & 2010 & 2015 & 2018 & $\begin{array}{l}2019 \\
\text { (Soll) }\end{array}$ & $\begin{array}{l}2020 \\
\text { (Soll) }\end{array}$ \\
\hline $\begin{array}{l}\text { Kieler Subventions- } \\
\text { bericht }\end{array}$ & 37,7 & 50,2 & 45 & 54,3 & 59,3 & 63,8 \\
\hline $\begin{array}{l}\text { Subventionsbericht } \\
\text { der Bundesregierung }\end{array}$ & 10,1 & 7 & 6,4 & 6,7 & 10,5 & 14,4 \\
\hline Anteil ${ }^{1}$ in $\%$ & 26,80 & 13,90 & 14,20 & 12,40 & 17,70 & 22,60 \\
\hline
\end{tabular}

werden. Das staatliche Angebot hat in diesen Fällen sozialpolitische Gründe (Zugang zu Bildung) oder soll gesellschaftlich erwünschte Zusatznutzen (externe Effekte wie z. B. hohes Bildungsniveau als Voraussetzung für den Produktionsfaktor Humankapital) sichern.

In diesen Überlegungen spiegeln sich nicht nur Abgrenzungsschwierigkeiten, sondern auch Auffassungsunterschiede wider, weil es eine Frage der politischen Bewertung ist, welche Zahlungen allgemeinen Staatsaufgaben (oder der Daseinsvorsorge) dienen oder ein öffentliches Angebot darstellen, das auch privatwirtschaftlich erfolgen könnte und deshalb - so die implizite These der Kieler Abgrenzung - privatwirtschaftlich angeboten werden sollte. Aus den Definitionen ergeben sich erhebliche Unterschiede im Subventionsvolumen. Die Kieler Definition weist z. B. für das Jahr 2018 54,3 Mio. Euro Finanzhilfen des Bundes (einschließlich Sonderhaushalte) aus, dieser Größe stehen 6,7 Mio. Euro Finanzhilfen des Bundes laut Subventionsbericht gegenüber.

\section{Arten und Ziele von Subventionen}

Mit den Ausgaben bzw. Einnahmeverzichten aufgrund von Steuervergünstigungen werden unterschiedliche Ziele verfolgt. Beispielsweise sollen Forschungsaktivitäten gefördert werden, Anpassungsprozesse im Strukturwandel erleichtert oder regionalpolitische Ziele verfolgt werden. Steuervergünstigungen werden vielfach damit gerechtfertigt, dass nationale Steuerbelastungen die internationale Wettbewerbsfähigkeit gefährden können. Zudem sind viele Subventionen Instrumente der Industrie- und Innovationspolitik, von der zunehmend erwartet wird, dass sie gemeinsam mit anderen wirtschaftspolitischen Handlungsfeldern Impulse zur nachhaltigen Umgestaltung von Produktions- und Konsumprozessen gibt und einen nachhaltigeren Strukturwandel vorantreibt (Kattel et al., 2020, 757). 
Es besteht die Gefahr, dass Subventionen den Wettbewerb zulasten nichtsubventionierter Akteure verzerren, denn je nach Ausgestaltung wirken sie mehr oder weniger selektiv. Vielfach wird angestrebt, Subventionen so zu gestalten, dass sie Investitionen beleben, die geeignet sind „Herausforderungen wie die Klimakatastrophe zu bewältigen" (Kattel et al., 2020, 759). Der Staat soll als Investor dazu beitragen, „,neue Arten von Wachstum zu katalysieren und auf diese Weise Investitionen und Innovationen des privaten Sektors anzuregen, was Erwartungen an zukünftige Wachstumsbereiche weckt" (Kattel et al., 2020, 759). So könnte der Staat durch strategische Investitionen in Hinblick auf klar definierte Ziele gesamtwirtschaftlich notwendige und sinnvolle Wachstumsrichtungen beeinflussen. Es sollen unterschiedliche Maßnahmen sektorenübergreifend koordiniert werden, sodass „neue Industrielandschaften“ (Kattel et al., 2020, 759) privatwirtschaftlich weiterentwickelt werden können. Diese Überlegungen gehen auf die Vorstellung zurück, dass staatliche und private Akteure und Investitionen gemeinsam ein vernetztes Innovationssystem bilden, das erforderlich ist, um vor allem risikoreiche Zukunftsinvestitionen zu ermöglichen, die privatwirtschaftlich nicht finanzierbar wären. In diesem Zusammenhang wird häufig darauf hingewiesen, dass vor allem in den USA wichtige Investitionen mit weitreichenden Spill-over-Effekten aus staatlichen Impulsen resultierten.

Dem Ziel, durch Subventionen Investitionen zu beleben, die den Strukturwandel stärken, wird im Subventionssystem zumindest ansatzweise Rechnung getragen. Angesichts der großen Zahl von Einzelmaßnahmen ist es allerdings schwierig, die Gesamtwirkungen aller Subventionen auf die Produktions- und Nachfragestruktur zu analysieren.

$\S 12$ des StabG schreibt vor, dass der Bund regelmäßig offenlegen muss, wie stark der Bundeshaushalt durch Subventionen belastet wird und wie sich Finanzhilfen und Steuervergünstigungen langfristig entwickeln werden. Neben den geplanten Volumina müssen auch Ziele von Subventionen, der Zeitpunkt des Auslaufens und verfügbare Evaluationen ausgewiesen werden. Damit können die Erwartungen der Wirtschaftssubjekte in Hinblick auf die öffentlichen Haushalte stabilisiert werden, zudem sollen Wirkungen auf den Strukturwandel verdeutlicht werden.

Unterschieden werden Erhaltungs-, Anpassungs- und Produktivitätshilfen, auch wenn die Einordnung konkreter Maßnahmen in diese Gruppen (vgl. Anlage 7 des Strukturberichts) nicht immer einfach ist (vgl. Tabelle 3). Erhaltungshilfen sollen notwendige Umstrukturierungen in Krisenunternehmen oder -branchen (vorübergehend) zeitlich strecken und so vermeiden, dass Schrumpfungsprozesse sich verstärken. Dies ist vor allem sinnvoll, wenn
Tabelle 3

Struktur des Subventionsvolumens nach

Subventionsarten

Anteile an den Finanzhilfen bzw. Steuervergünstigungen des Bundes in \%

$$
20192020 \quad 2021 \quad 2022
$$

\section{Finanzhilfen ${ }^{1}$}

\begin{tabular}{lllll}
\hline für Betriebe oder Wirtschaftszweige & 68,9 & 76,7 & 79 & 74,8 \\
\hline
\end{tabular}

davon:

\begin{tabular}{lrrrr}
\hline Erhaltungshilfen & 4,1 & 4,0 & 6,0 & 1,6 \\
\hline Anpassungshilfen & 34,2 & 42,2 & 28,7 & 25,1 \\
\hline Produktivitäts-/Wachstumshilfen & 12,3 & 9,5 & 17,0 & 17,7 \\
\hline \multicolumn{1}{c}{ Sonstige Finanzhilfen } & 18,4 & 21,0 & 27,4 & 30,5 \\
\hline für private Haushalte ${ }^{2}$ & 31,1 & 23,3 & 21,0 & 25,2 \\
\hline Summe Finanzhilfen $^{\prime}$ & 100 & 100 & 100 & 100
\end{tabular}

Steuervergünstigungen

\begin{tabular}{lllll}
\hline für Betriebe oder Wirtschaftszweige $\quad 92,6$ & 91,7 & 92,3 & 92,9 \\
\hline
\end{tabular}

davon:

\begin{tabular}{lrrrr}
\hline Erhaltungshilfen & 46,4 & 47,0 & 47,1 & 45,6 \\
\hline Anpassungshilfen & 6,8 & 7,5 & 12,0 & 12,5 \\
\hline Produktivitäts-/Wachstumshilfen & 15,8 & 18,1 & 16,2 & 15,5 \\
\hline Sonstige Steuervergünstigungen & 23,5 & 19,1 & 17,1 & 19,2 \\
\hline für private Haushalte ${ }^{2}$ & 7,4 & 8,3 & 7,7 & 7,1 \\
\hline Summe Steuervergünstigungen $^{\prime}$ & 100 & 100 & 100 & 100 \\
\hline
\end{tabular}

Finanzhilfen und Steuervergünstigungen des Bundes insgesamt

\begin{tabular}{lllll}
\hline für Betriebe oder Wirtschaftszweige $\quad 84,6$ & 85,2 & 84,6 & 82,3
\end{tabular}

davon:

\begin{tabular}{lrrrr}
\hline Erhaltungshilfen & 32,1 & 28,4 & 23,3 & 19,8 \\
\hline Anpassungshilfen & 16,1 & 22,5 & 21,6 & 19,9 \\
\hline $\begin{array}{l}\text { Produktivitäts-/Wachstumshilfen } \\
\text { Sonstige Finanzhilfen und Steuerver- } \\
\text { günstigungen }\end{array}$ & 14,6 & 14,4 & 16,6 & 16,8 \\
\hline $\begin{array}{l}\text { für private Haushalte } \\
2\end{array}$ & 15,4 & 14,8 & 15,4 & 17,7 \\
\hline Summe insgesamt $^{\text {Sum }}$ & 100 & 100 & 100 & 100 \\
\hline
\end{tabular}

${ }^{1}$ Für die Jahre 2019 und 2020 Ist-Werte; für 2021, 2022 Soll-Werte. ${ }^{2}$ Betriebe und Wirtschaftszweige werden mittelbar begünstigt.

Quelle: Für das Jahr 2017 27. Subventionsbericht (BMF, 2019, 40) sowie für die Jahre 2020, 2021 28. Subventionsbericht (BMF, 2021, 48).

Krisenbranchen regional konzentriert sind, sodass massive Arbeitsplatzverluste negative Multiplikatorwirkungen auslösen könnten. Erhaltungshilfen machten 2019 etwa $32 \%$ des gesamten Subventionsvolumens des Bundes aus (vgl. Tabelle 3). Seit 2019 geht der Anteil allerdings deutlich zurück, auch wenn er vor allem bei den Steuererleichterungen mit $46 \%$ weiterhin sehr hoch ist. Anpassungshilfen unterstützen im Zuge des Strukturwandels erforderliche Anpassungsprozesse in Unternehmen (2019 
ca. $16 \%)$. In diese Rubrik gehören z.B. die Ausgaben im Bereich der Gemeinschaftsaufgabe „Verbesserung der regionalen Wirtschaftsstruktur" (GRW) (Anlage 7, Nr. 40), sowie die Gewährung von Anpassungsgeld (APG) an Beschäftigte des Steinkohlenbergbaus (Anlage 7, Nr. 14). Ihr Anteil nahm in den letzten Jahren zu. Produktivitätshilfen fördern die Wettbewerbsfähigkeit von Unternehmen oder Branchen (2019 knapp 15\%). Hierzu gehören z.B. die Subventionen aus dem Zentralen Innovationsprogramm Mittelstand (ZIM) (Anlage 7, Nr. 30). Ergänzend werden sonstige Hilfen für Unternehmen (2019 ca. $22 \%$ ) ausgewiesen. Dies umfasst z.B. die Förderung von Einzelmaßnahmen zur Nutzung erneuerbarer Energien, Maßnahmen zur Steigerung der Energieeffizienz (Anlage 7, Nr. 18 und Nr. 15, 16). Hilfen für private Haushalte (z.B. Förderung von Maßnahmen zur energetischen Gebäudesanierung (Anlage 7, Nr. 87) und die Prämien nach dem Wohnungsbau-Prämiengesetz (Anlage 7, Nr. 93) (2019 ca. 15\%) werden zusätzlich ausgewiesen.

Da im Rahmen der Strukturpolitik auf Dauer gezahlte Erhaltungshilfen weniger sinnvoll sind als Anpassungs- und Produktivitätshilfen, ist insbesondere die Struktur der Steuervergünstigungen finanzpolitisch immer noch bedenklich. Zudem übersteigen die Steuervergünstigungen die Finanzhilfen deutlich, obwohl Finanzhilfen als geeigneter gelten: Die Höhe von Einnahmeverzichten bei den Steuereinnahmen ist vorab nicht limitiert.

Außerdem werden Steuererleichterungen nicht in den jährlichen Haushaltsverhandlungen festgelegt und schnell als reguläres Steuerrecht wahrgenommen. Sie sind schwerer aufzuheben, nutzen nur Unternehmen, die Steuern zahlen und begünstigen Mitnahmeeffekte. Zudem werden bei den Gemeinschaftssteuern Einnahmeausfälle von Bund, Ländern und Gemeinden gemeinsam getragen. Das Steuerrecht kann daher häufig nur geändert werden, wenn der Bundesrat zustimmt. Sollen Steuervergünstigungen auf Finanzhilfen umgestellt werden, muss eine Gebietskörperschaft die Subvention finanzieren, wogegen Widerstände bestehen können (BMF, 2021, 15).

Knapp die Hälfte (ca. 49 \% im Jahr 2019) der Subventionen entfällt auf die Gewerbliche Wirtschaft, beispielsweise im Rahmen von Fördermaßnahmen in den Bereichen rationelle Energieverwendung oder alternative Formen der erneuerbaren Energien. Außerdem werden jeweils ca. $11 \%$ bzw. $12 \%$ der Subventionen für die Bereiche Wohnungswesen und Verkehr aufgewendet, beispielsweise durch Baukindergeld und Zahlungen zur „Reduzierung der Trassenpreise im Schienengüterverkehr". Die letztgenannte Maßnahme soll die Wettbewerbsfähigkeit des Schienengüterverkehrs verbessern, sie ist im Subventionsbericht als Erhaltungssubvention klassifiziert (vgl. 28. Subventionsbericht, BMF, 2021, 9 sowie Anlage 7, Nr. 96).

\section{Subventionen - Instrumente einer gestaltenden Strukturpolitik?}

Notwendigkeit und Ausgestaltung einzelner Subventionsprogramme und grundsätzliche Argumente für und gegen Subventionen werden immer wieder erörtert: Subventionen sind ordnungs- und wettbewerbspolitisch umstritten, weil subventionierte Unternehmen nicht ihre gesamten Produktionskosten aus Markterlösen decken müssen. Damit werden die relativen Preise und die Wirkung der Preisfunktionen (Informations-, Lenkungs- und Anreizfunktion) verändert, die Selektionsfunktion des Wettbewerbs beeinträchtigt und möglicherweise Produktionsfaktoren in nichtwettbewerbsfähigen Bereichen gebunden. Aus strukturpolitischen Gründen werden allerdings häufig nicht einzelne Unternehmen, sondern unternehmerische Aktivitäten gefördert, die im gesamtgesellschaftlichen Interesse liegen. Beispielsweise können Forschungen in bestimmten Bereichen unterstützt werden, die wegen des hohen Kapitalaufwands und des hohen Risikos aus dem Marktprozess heraus nicht oder nicht schnell genug vorangetrieben werden.

Subventionen mildern Anpassungslasten, wenn Grenzanbieter vorübergehend Zeit zur Wiederherstellung ihrer Wettbewerbsfähigkeit gewinnen. Dies erfordert allerdings eine angemessene Befristung und eine degressive Ausgestaltung der Subventionen, damit eine allmähliche Anpassung an die Marktsituation möglich, aber auch zwingend erforderlich ist. Vor allem im Zusammenhang mit Erhaltungssubventionen wird bemängelt, dass der Anreiz zur Umstrukturierung in subventionierten Unternehmen abnehmen kann und stattdessen immer wieder um staatliche Unterstützung zugunsten von einflussreichen Interessengruppen geworben wird (Rent-Seeking-Verhalten). Politisch motivierte Subventionen gehen möglicherweise nicht mit langfristig tragfähigen Konzepten zur Umstrukturierung einher. Regional dominierende Großunternehmen, Gewerkschaften oder Regionalpolitiker:innen könnten zu diesem Verhalten neigen, wenn es nicht gelingt, Subventionen glaubwürdig und verbindlich zu befristen.

Wenn Subventionen die notwendige Zeit für erfolgreiche Umstrukturierungen schaffen, können sie dazu beitragen, Unternehmen und Arbeitsplätze zu sichern und verhindern, dass sich - vor allem in einzelnen Regionen - Anpassungsprozesse krisenhaft verschärfen. Allerdings belasten die Zahlungen die öffentlichen Haushalte und diejenigen, die die Mittel dafür aufbringen müssen. Daher wird befürchtet, dass öffentliche und private Investitionen zurückgehen könnten, was mit negativen Wachstumswirkungen einhergehen würde. 
Trotz aller Bedenken werden häufig neue Subventionen eingeführt, deren (normativ begründete) Ziele sich nicht immer ökonomisch beurteilen lassen. Im Subventionsbericht werden im Wesentlichen beschäftigungs-, verteilungs- und umweltpolitische Begründungen angeführt, es wird also auf langfristige und gesellschaftlich sinnvolle Ziele verwiesen, die aus dem Marktprozess heraus nicht oder nicht schnell genug verfolgt werden. Viele Subventionen sollen z.B. die Entwicklung klimafreundlicher Technologien fördern. Damit greift die Bundesregierung implizit auf Instrumente einer gestaltenden Strukturpolitik zurück, die mit Marktversagen angesichts gesellschaftlicher Herausforderungen begründet werden können. „Neben dem Klimaschutz als ein politisches Kernanliegen liegen weitere aktuelle Förderschwerpunkte in den Bereichen Wohnungsbau, Digitalisierung und Mobilität. Durch eine klare inhaltliche Schwerpunktsetzung in prioritären Bereichen werden mit subventionspolitischen Maßnahmen wichtige Impulse gegeben, um die Zukunfts- und Innovationsfähigkeit der Wirtschaft zu stärken, die wirtschaftlichen Folgen der Pandemie zukunftsorientiert zu bewältigen, Klima- und Umweltschutz voranzubringen und sozialen Ausgleich zu fördern“ (BMF, 2021, 7). Allerdings trägt eine solche Begründung nur, wenn staatliche Maßnahmen zu besseren Ergebnissen führen als die Marktsteuerung. Daher ist Begleitforschung, die sich an Leitlinien der Subventionsvergabe orientiert, sinnvoll. Auf diese Weise können die Effektivität und die Effizienz von Subventionen beurteilt und ihr Beitrag zur Nachhaltigkeitsstrategie geprüft werden.

\section{Leitlinien der Subventionsvergabe}

Im 19. Subventionsbericht (2003) sind Leitlinien der Subventionierung formuliert worden, die seitdem im Kern unverändert sind, es wurde allerdings eine Nachhaltigkeitsprüfung ergänzt (2015). Anhand dieser Leitlinien werden Notwendigkeit und Ausgestaltung der Subventionen überprüft. Zudem werden Evaluationen gefordert, deren Ergebnisse in den Anhängen des Subventionsberichts für jede Maßnahme kurz umrissen werden.

Anhand der Leitlinien lassen sich einige Merkmale der Subventionen des Bundes aufzeigen: Zwischen 2017 und 2020 sind die Finanzhilfen stärker gestiegen als die Steuervergünstigungen, daher ist der Anteil der Steuervergünstigungen an den Subventionen zwischen 2017 und 2020 zwar von ca. $72 \%$ auf $57 \%$ zurückgegangen (BMF, 2019, 6; und BMF, 2021, 21), aber immer noch hoch.

Um konservierende Strukturpolitik zu vermeiden, wird neben der Befristung auch gefordert, dass Subventionen degressiv ausgestaltet sein sollen. Diese Forderung ist allerdings nicht leicht umzusetzen: Für die richtige Be- fristung müssten Politiker:innen in der Lage sein, einzuschätzen, ob Unternehmen sich erfolgreich umstrukturieren können und wie viel Zeit und Unterstützung sie dafür benötigen. Dies setzt Marktkenntnisse und Informationen voraus, welche die Unternehmen möglicherweise nicht weitergeben wollen, damit Subventionen weitergezahlt werden. Staatliche Informationsdefizite können daher bewirken, dass Subventionen zu lange fortgeführt werden. Aber auch die Verkürzung von Subventionszeiten kann Risiken mit sich bringen: Werden Subventionen in zu geringem Umfang und zu kurz gewährt, reichen die Maßnahmen unter Umständen nicht, um die Wettbewerbsfähigkeit wiederherzustellen oder den notwendigen Strukturwandel zu fördern.

Laut Subventionsbericht sind rund $76 \%$ der Finanzhilfen befristet, gemessen am Subventionsvolumen sogar fast $90 \%$ (BMF, 2021, 67). Allerdings konnten nur ca. $16 \%$ der Finanzhilfen (bzw. knapp $19 \%$ des Volumens) degressiv gestaltet werden (BMF, 2021, 68). Von den aktuellen Finanzhilfen sind nur 13 gleichzeitig befristet und degressiv gestaltet (ca. $11 \%$ des Gesamtvolumens der Finanzhilfen) (BMF, 2021, 69). Bei den Steuervergünstigungen, die auf gesetzlichen Regelungen beruhen, ist eine degressive Gestaltung „selten möglich“ (BMF, 2021, 68) - hier sind lediglich $26 \%$ der Maßnahmen mit einem Volumen von knapp $34 \%$ der geschätzten Steuervergünstigungen (BMF, 2021, 67) befristet.

Zusätzlich soll nach den Leitlinien das Subventionsvolumen gedeckelt werden, d.h. neue Maßnahmen können nur eingeführt werden, wenn alte auslaufen. Die langfristige Entwicklung der Subventionsquoten zeigt, dass erst seit 2020 ein Anstieg der Subventionsquote zu erkennen ist, der zumindest teilweise auf Corona-Maßnahmen zurückgeht. Insbesondere der Anteil der Subventionen am Bruttoinlandsprodukt ist nominal bis 2019 weitgehend konstant (vgl. Abbildung 1) und der Anteil der Steuervergünstigungen an den Steuereinnahmen konnte zwischen 2009 und 2019 reduziert werden. Allerdings ist der Anteil der Steuervergünstigungen an den gesamten Subventionen - anders als in den Leitlinien gefordert - hoch, sodass das Ziel, Subventionen verstärkt in Form von Finanzhilfen zu zahlen, nicht umfassend erreicht wird.

\section{Subventionskontrolle und Möglichkeiten von Subventionskürzungen}

Die regelmäßig zu veröffentlichenden Subventionsberichte tragen zur Transparenz der Subventionslandschaft bei und zwingen zur Rechtfertigung der einzelnen Maßnahmen entsprechend den Leitlinien. Zudem werden immer wieder einzelne Maßnahmen evaluiert. Im Blickpunkt der Evaluation von Subventionen stehen ihre Auswirkungen 


\section{Abbildung 1}

\section{Subventionsquoten 2000 bis 2020}

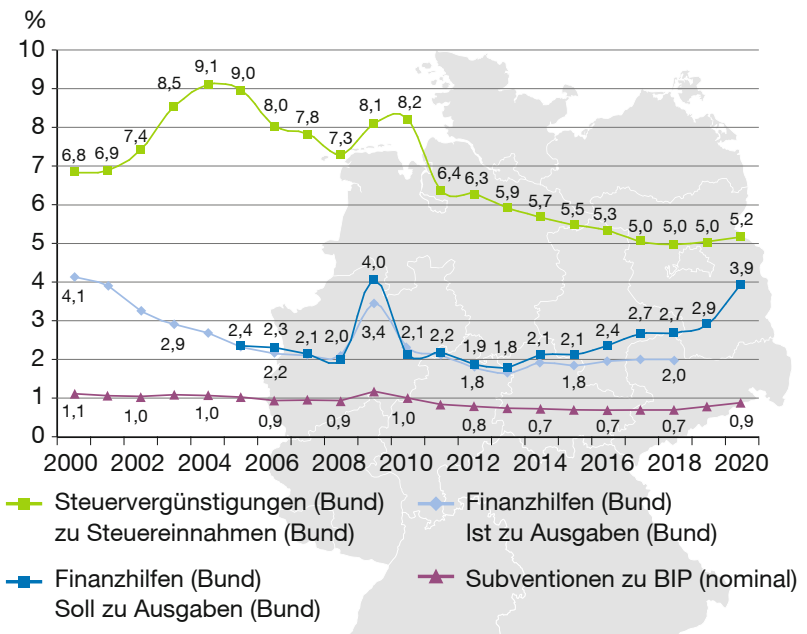

Quelle: https://www.bundesfinanzministerium.de/Monatsberichte/2019/11/ Bilder/B02-Abb03.jpg?__blob=poster\&v=4 (24. November 2021).

auf die nationale und internationale Wettbewerbssituation sowie eventuelle Gewöhnungs- und Mitnahmeeffekte. Erforderlich ist eine allokative Prüfung (Notwendigkeit der Subvention), eine instrumentelle Prüfung (geeignete Subventionsform ohne Nebenwirkungen, wie z.B. Mitnahmeeffekte) und eine operative Subventionskontrolle (kostengünstige Maßnahmen) (Thöne und Happ, 2015, 102). Im aktuellen Subventionsbericht weist die Bundesregierung darauf hin, dass 72 von 128 Finanzhilfen intern oder extern evaluiert wurden. Dies gilt auch für 46 von 106 Steuervergünstigungen. Allerdings entsteht wegen der Vielzahl der Maßnahmen dadurch noch kein Gesamtbild über die Wirkungen der Subventionen, die zum Teil sehr unterschiedliche Ziele verfolgen.

Seit der Neufassung der Leitlinien im Jahr 2015 soll zusätzlich eine Prüfung der Nachhaltigkeit der Subventionen erfolgen, dabei werden die Maßnahmen anhand der Deutschen Nachhaltigkeitsstrategie (Bundesregierung, 2020) bewertet. Hier sollen die ökonomischen, sozialen und ökologischen Wirkungen der einzelnen Subventionen geprüft werden. ${ }^{1}$ Subventionen sollen zur Generationengerechtigkeit beitragen, sozialen Zusammenhalt und Lebensqualität fördern und international verantwortbar sein (BMF, 2021, 74). Besonders betont wird der Beitrag der Subventionen zum Klimaschutz und zur Ressourcenschonung sowie zur Sicherung der Beschäftigung. Diese Prüfung erfolgt allerdings noch eher qualitativ durch die fachlich zuständigen Ministerien. Die Bundesregierung

1 Die Ergebnisse dieser Prüfungen werden für jede Maßnahme in den Anhängen 7 und 8 des Subventionsberichts dargestellt. weist darauf hin, dass 67 der 128 Finanzhilfen (Finanzvolumen ca. 16 Mrd. Euro) einen positiven Bezug zu den in der Deutschen Nachhaltigkeitsstrategie verankerten Umwelt- und Klimaschutzzielen aufweisen (BMF, 2021, 6). Zugleich werden Zielkonflikte erkennbar. „Subventionen führen tendenziell zu einem erhöhten Verbrauch an finanziellen, materiellen und/oder natürlichen Ressourcen“ (BMF, 2021, 75). Insgesamt positiv bewertet die Bundesregierung vor allem neu eingeführte Finanzhilfen (BMF, 2021, 78).

Bei der „Durchforstung“ der komplexen Subventionslandschaft sollten strukturpolitische Ziele auch in Zukunft nicht vernachlässigt werden. In diesem Zusammenhang ist es sinnvoll, ein klares Zielsystem zu formulieren und Zielkonflikte offenzulegen. Die Ziele einer missionsorientierten Strukturpolitik können hier als Messlatte dienen. Daher werden vor allem umweltschädliche Subventionen immer wieder kritisiert. Vorschläge zur Kürzung umweltschädlicher Subventionen wurden z. B. vom Umweltbundesamt gemacht (Umweltbundesamt, 2021).

Vorgesehene Subventionskürzungen müssen Evaluationsergebnisse berücksichtigen und die angesprochenen Subventionsziele auf ihre Vereinbarkeit mit den Zielen bzw. Missionen der Strukturpolitik beachten. Dabei sind verschiedene Vorgehensweisen denkbar: Durch zeitliche Befristung und einen degressiven Verlauf sollen Dauersubventionen für einzelne Unternehmen oder Branchen verhindert und Spielräume für zukunftsträchtige Fördermaßnahmen geschaffen werden. Allerdings stößt die Streichung einzelner Subventionen immer wieder auf Widerstände der Begünstigten. Ein Beispiel stellt die Diskussion darüber dar, welche Güter mit dem reduzierten Mehrwertsteuersatz versteuert werden sollen. Obwohl die Auswahl der ermäßigt besteuerten Güter nicht durchgängig damit begründet werden kann, dass die regressive Wirkung der Mehrwertsteuer gemildert werden soll, gab es in der Vergangenheit kaum Korrekturen, obwohl dazu mehrere Evaluationsstudien und Vorschläge vorliegen (Kambeck und Wiegard, 2011).

Diskutiert wird auch die Privatisierung öffentlich angebotener Leistungen, sodass staatliche Angebote in Bereichen gekürzt werden, die auch privatwirtschaftlich organisiert werden könnten. Beispielsweise werden Teile des staatlichen Kultur-, Bildungs- oder Gesundheitsbereichs in Frage gestellt, die vom Kieler Institut für Weltwirtschaft als originär marktwirtschaftlich angesehen werden. Die Folge wäre aber wohl, dass diese Angebote primär nach Auslastungskriterien, nicht mehr flächendeckend und darüber hinaus zu höheren Preisen angeboten würden, sodass sie nicht mehr in allen Regionen und für alle zugänglich wären. Es ist eine Frage der politischen Ent- 
scheidung, ob und in welchem Umfang Kürzungen dieser staatlichen Angebote sozial nachhaltig, politisch vertretbar und mehrheitsfähig wären.

\section{Schlussbemerkung}

Das Subventionssystem ist - vor allem wegen der vielen Maßnahmen - intransparent, bürokratisch aufwändig und nicht widerspruchsfrei. Es erfültt auch nicht alle Leitlinien der Subventionierung, auch wenn diese regelmäßig überprüft werden. Trotzdem ist ein Teil der Subventionen dazu geeignet, Ziele einer gestaltenden Strukturpolitik zu erfüllen. Beispielsweise dient ein erheblicher Teil der Subventionen der Forschungsförderung, vor allem in den Bereichen Digitalisierung, Energie- und Klimapolitik - insofern ist eine Ausrichtung auf Zukunftsaufgaben erkennbar. Kattel et al. sehen z. B. die Maßnahmen im Zusammenhang mit der Energiewende als positives Beispiel an (Kattel et al., 2020, 760). Zudem dominieren, zumindest bei den Finanzhilfen die Anpassungs- und Produktivitätshilfen. Bei den Steuervergünstigungen, die im Gegensatz zur Maßgabe der Leitlinien der Subventionierung ohnehin noch sehr umfangreich sind, handelt es sich vielfach um unbefristete Erhaltungssubventionen. Hier wäre eine Umstrukturierung wünschenswert, auch wenn dies politisch schwer umzusetzen ist.

Strukturpolitisch begründete Subventionen sollen in erster Linie unternehmens- oder branchenspezifisch wirken, darüber hinaus sollen sie notwendige Forschungs- und Entwicklungsrichtungen im Strukturwandel stärken, bei denen Marktversagen vermutet werden kann. Staatliche Schwerpunktsetzung soll private Investitionen unterstützen, die gesamtgesellschaftlichen Zielen dienen. Im Zuge einer missionsorientierten Strukturpolitik ist allerdings eine klare Zielsetzung erforderlich. Angesichts des erheblichen industriellen Transformationsbedarfs sind Impulse notwendig, die Forschung und Investitionen in Hinblick auf nachhaltigere Produktions- und Konsumstrukturen anregen. Um die Subventionslandschaft etwas zu lichten, ist es sinnvoll, vor allem umweltschädliche Subventionen abzubauen. Zudem ist die Nachhaltigkeitsbewertung der Subventionen im Subventionsbericht ein erster, aber ausbaufähiger Schritt in diese Richtung. Insofern sind Subventionskontrolle, aber auch die politische Einigung auf prioritäre Ziele, permanente Aufgaben.

\section{Literatur}

Boss, A. und A. Rosenschon (2006), Der Kieler Subventionsbericht: Grundlagen, Ergebnisse, Schlussfolgerungen, Kieler Diskussionsbeiträge, 423, Institut für Weltwirtschaft (IfW).

Bundesfinanzministerium (BMF) (2019), Bericht der Bundesregierung über die Entwicklung der Finanzhilfen des Bundes und der Steuervergünstigungen für die Jahre 2017-2020, https://www.bundesfinanzministerium.de/Content/DE/Downloads/Broschueren_Bestellservice/202003-01-Subventionsbericht.pdf (9. November 2021).

Bundesfinanzministerium (BMF) (2021), Bericht der Bundesregierung über die Entwicklung der Finanzhilfen des Bundes und der Steuervergünstigungen für die Jahre 2019-2022, https://www. bundesfinanzministerium.de/Content/DE/Pressemitteilungen/ Finanzpolitik/2021/08/2021-08-18-zukunftsorientierte-subventionspolitik-bundesregierung.html (2. November 2021).

Bundesregierung (2020), Deutsche Nachhaltigkeitsstrategie - Weiterentwicklung 2021, https://www.bundesregierung.de/breg-de/themen/ nachhaltigkeitspolitik/eine-strategie-begleitet-uns/die-deutschenachhaltigkeitsstrategie, https://www.bundesregierung.de/breg-de/ aktuelles/nachhaltigkeitsstrategie-2021-1873560 (13. November 2021).

Kambeck, R. und W. Wiegard (2011), Analyse der fiskalischen Auswirkungen des ermäßigten Umsatzsteuersatzes in Deutschland unter Verwendung eines Simulationsmodells sowie der Wachstumseffekte von Straffungseffekten.

Kattel, R., M. Mazzucato, K. Haverkamp und J. Ryan-Collins (2020), Industriestrategie der nächsten Generation für Deutschland, Wirtschaftsdienst, 100(10), 757-762, https://www.wirtschaftsdienst.eu/ inhalt/jahr/2020/heft/10/beitrag/industriestrategie-der-naechstengeneration-fuer-deutschland.html (24. November 2021).

Laaser, C. F. und A. Rosenschon (2020), Kieler Subventionsbericht 2020. Subventionen auf dem Vormarsch, Kieler Beiträge zur Wirtschaftspolitik, 29, Institut für Weltwirtschaft (IfW).

Sachverständigenrat zur Begutachtung der gesamtwirtschaftlichen Entwicklung 2019/2020: Den Strukturwandel meistern, Kapitel 3: Industriepolitik: Strukturwandel als Chance, 140-197, Zf. 245 ff.

Thöne, M. und D. Happ (2015), Institutionelle Strukturen zur Verbesserung von Transparenz und Wirksamkeit von Subventionen, FiFo-Berichte, 17, Finanzwissenschaftliches Forschungsinstitut an der Universität zu Köln (FiFo Köln), Köln, https://kups.ub.uni-koeln.de/6209/ (13. November 2021).

Umweltbundesamt (2021), Umweltschädliche Subventionen in Deutschland, aktualisierte Ausgabe 2021, Berlin, https://www.umweltbundesamt.de/publikationen/ umweltschaedliche-subventionen-in-deutschland-2016 (14. November 2021).

Title: Measures of a Mission-Oriented Structural Policy - Considerations to the $28^{\text {th }}$ Report on German Subsidies

Abstract: The landscape of German subsidies is manifold. The regular report about subsidies contains information about aims, time limits and evaluations of each financial aid and tax relief plan. The article details some features of German subsidies with regard to aims (conservation, adaptation and increased raise of productivity) and to politically defined guidelines on subsidies. Although the subsidies do not comply with these guidelines completely, some of them can be seen as instruments of a mission-oriented structural policy. However, clear definitions of social aims of subsidies, evaluation and an improved evaluation of sustainability remain to be permanent tasks. 